\title{
ERRADICAÇÃO DE Fusarium oxysporum EM SEMENTES DE ALFAFA UTILIZANDO TERMO E QUIMIOTERAPIA
}

\author{
MARTA. A. S. MENDES ${ }^{1}$, PAULA M. M. P. LIMA*, JOSÉ N. L. FONSECA ${ }^{1}$ \& MARIA F. SANTOS* \\ ${ }^{1}$ Laboratório de Quarentena Vegetal, Embrapa Recursos Genéticos e Biotecnologia (CENARGEN), Empresa Brasileira de \\ Pesquisa Agropecuária, Cx. Postal 02372, CEP 70770-900 Brasília, DF, fax: (061) 448-4627, \\ e-mail: martamen@ cenargen.embrapa.br
}

(Aceito para publicação em 11/04/2001)

Autor para correspondência: Marta A. S. Mendes

MENDES, M.A.S., LIMA, P.M.M.P., FONSECA, J.N.L. \& SANTOS, M.F. Erradicação de Fusarium oxysporum em sementes de alfafa utilizando termo e quimioterapia. Fitopatologia Brasileira 26:148-152. 2001.

\section{RESUMO}

Sementes de alfafa (Medicago sativa) contaminadas in vitro com Fusarium oxysporum foram submetidas a tratamento térmico seco, térmico úmido e químico, visando a erradicação deste patógeno. O tratamento térmico seco foi realizado em câmara com fluxo de ar constante, em sementes com umidade relativa abaixo de $5 \%$, pré-tratamento a $60{ }^{\circ} \mathrm{C}$ por 3 ou $6 \mathrm{~h}$, seguido do tratamento a $90{ }^{\circ} \mathrm{C}$ por 3 ou $6 \mathrm{~h}$. O tratamento térmico úmido foi realizado em banho-maria, com pré-tratamento a $40{ }^{\circ} \mathrm{C}$ por 10,20 ou 30 min e tratamentos a $50{ }^{\circ} \mathrm{C}$ ou $60{ }^{\circ} \mathrm{C}$ por 10,20 e $30 \mathrm{~min}$. Os produtos químicos testados foram benomyl, thiabendazole, iprodione e thiram, nas dosagens recomendadas pelos fabricantes e o triplo dessas dosagens. Foram avaliados os efeitos dos tratamentos no controle de F. oxysporum, pelo método de "Blotter test", e na germinação das sementes. O tratamento térmico úmido, $40^{\circ} \mathrm{C}$ por $20 \mathrm{~min}$, seguido de 50 ${ }^{\circ} \mathrm{C}$ por $20 \mathrm{~min}$, erradicou o patógeno $F$. oxysporum, mas reduziu significativamente a germinação das sementes. $\mathrm{O}$ tratamento térmico seco a $60^{\circ} \mathrm{C}$ por $3 \mathrm{~h}$, seguido de $90{ }^{\circ} \mathrm{C}$ por 3 ou 6 h de exposição, erradicou $F$. oxysporum sem alterar significativamente a germinação das sementes. Benomyl foi o mais eficiente dos produtos químicos testados, erradicando F. oxysporum sem afetar a germinação das sementes de alfafa.

Palavras-chave: tratamento térmico seco, tratamento térmico úmido, tratamento químico, fungo, semente.

\section{ABSTRACT \\ Eradication of Fusarium oxysporum in alfalfa seeds by thermal and chemical treatments}

The dry thermal treatment was conducted in a continuous air flux chamber on alfafa (Medicago sativa) seeds with relative humidity under $5 \%$. Seeds underwent a pre-treatment for $60{ }^{\circ} \mathrm{C}$ during three or six hours, followed by a treatment at $90{ }^{\circ} \mathrm{C}$ for three or six hours. The wet treatment was conducted in water bath, and consisted of a pre-treatment at $40{ }^{\circ} \mathrm{C}$ for 10,20 or $30 \mathrm{~min}$, and then treatments at $50{ }^{\circ} \mathrm{C}$ or $60{ }^{\circ} \mathrm{C}$ for 10,20 or $30 \mathrm{~min}$. The chemicals tested were benomyl, thiabendazole, iprodione and thiram, at the dosages recommended by their manufactures and also at three times these dosages. The wet thermal treatment at $40{ }^{\circ} \mathrm{C}$ for $20 \mathrm{~min}$, followed by the treatment at $50{ }^{\circ} \mathrm{C}$ for $20 \mathrm{~min}$, were effective in the eradication of Fusarium oxysporum, but significantly affected the alfafa seed germination. The dry thermal treatment at $60{ }^{\circ} \mathrm{C}$ for $3 \mathrm{~h}$, followed by the treatment of $90{ }^{\circ} \mathrm{C}$ for 3 to $6 \mathrm{~h}$, eradicated $F$. oxysporum without a significant reduction in seed germination. Benomyl was the most efficient chemical product in eradicating $F$. oxysporum without affecting the alfalfa seed germination.

\section{INTRODUÇÃO}

Em alfafa (Medicago sativa L.), o fungo Fusarium oxysporum Schlecht., transmitido por sementes, tem sido relatado como um dos principais agentes da podridão radicular, doença que reduz significativamente a produtividade dessa planta (Ellankaya et al., 1995). Segundo OrliezLuthardt (1998), os isolados de F. oxysporum que causam

\footnotetext{
* Bolsista da EMBRAPA Recursos Genéticos e Biotecnologia
}

murcha, obtidos de sementes, apresentam virulência maior do que os isolados obtidos de hastes de plantas doentes.

O controle de fungos transmitidos por sementes é necessário principalmente para se evitar a transmissão de patógenos a longas distâncias e também para melhorar a qualidade do material utilizado para propagação na mesma região. $\mathrm{O}$ emprego de agroquímicos tem sido a forma mais comum utilizada no controle de doenças, porém verificam-se algumas desvantagens, principalmente por contaminar o meio ambiente com resíduos tóxicos não biodegradáveis e pela resistência 
Erradicação de Fusarium oxysporum em sementes de alfafa utilizando termo...

desenvolvida pelos microrganismos a esses compostos. A aplicação de agentes de controle biológico em sementes, como isolados de Trichoderma Pers. (Kaur \& Mukhopadhyay, 1992; Reis et al., 1995), uso de formulações de Pseudomonas fluorescens (Trevisan) Migula (Vidhyasekaran \& Muthamilan, 1995) e o controle integrado através da aplicação de agentes microbianos e fungicidas reduziram a incidência de doenças causadas por F. oxysporum (Raju \& Sivaprakasam, 1989). A aplicação de óleo essencial de algumas plantas, como Nardostachys jatamansi Meier \& Al. em sementes inibiu o crescimento de F. oxysporum (Dhirendra et al., 1995).

A técnica de controle de fungos transmitidos por sementes utilizando calor seco ou úmido, tem apresentado resultados promissores e foi considerada viável nos procedimentos fitossanitários de quarentena (De Leon \& Grudloyma, 1994). Por meio desta terapia foram controlados e/ou erradicados diversos patógenos, entre eles Phoma betae Frank em sementes de beterraba (Beta vulgaris L.) (Bottcher \& Horn, 1992), Alternaria brassicae (Berk.) Sacc. em sementes de Brassica juncea Coss. (Randhawa \& Aulakh, 1984), Cercospora kikuchii (Matsumoto \& Tomoyasu) Gardner em sementes de soja [Glycine max (L.) Merril] (Fonseca et al., 1994) e Alternaria radicina Meier \& Al. em sementes de cenoura (Daucus carota L.) (Pryor et al., 1994). A termoterapia também reduziu consideravelmente os fungos que contaminavam superficialmente as sementes, aumentando desta forma, o seu poder germinativo (Venkatasubbaiah et al., 1984; Donald \& Lundquist, 1988) e vigor (Raju \& Sivaprakasan, 1989).

Este trabalho teve por objetivo desenvolver uma metodologia visando erradicar $F$. oxysporum de sementes de alfafa, utilizando termo ou quimioterapia.

\section{MATERIAL E MÉTODOS}

\section{Inoculação de sementes de alfafa com $\boldsymbol{F}$. oxysporum}

A partir de sementes de alfafa infetadas, foi obtido um isolado de $F$. oxysporum, sendo o mesmo cultivado em meio de cultura BDA (Batata-Dextrose-Ágar), sob luz fluorescente contínua, a temperatura de $28{ }^{\circ} \mathrm{C}$, por 10 dias. Decorrido esse período o fungo foi inoculado em sementes de alfafa, utilizandose para isso a metodologia de Garrido et al. (1996), com modificações, conforme descrito a seguir. As sementes de alfafa (60 g) foram colocadas em um bequer de $250 \mathrm{ml}$ e imersas em suspensão de $10^{8}$ conídios $/ \mathrm{ml}$, por $15 \mathrm{~min}$. Durante este tempo, as sementes foram agitadas com o auxílio de um bastão de vidro, de 3 em 3 min, para homogeneizar o inóculo. Após este procedimento as sementes foram filtradas em gaze e colocadas sobre duas folhas de papel de filtro, dentro de dessecador acoplado à bomba a vácuo $(200 \mathrm{mmHg})$, que permaneceu em funcionamento por $3 \mathrm{~h}$. As sementes foram mantidas no dessecador até serem submetidas aos tratamentos.

\section{Tratamento térmico a seco das sementes inoculadas com F. oxysporum}

As sementes de alfafa foram colocadas, previamente, em câmara de secagem com 16 \% UR (Umidade Relativa) e temperatura de $22{ }^{\circ} \mathrm{C}$, durante 15 dias, sendo depois submetidas a um pré-tratamento térmico a $60{ }^{\circ} \mathrm{C}$ por 3 e $6 \mathrm{~h}$, passando para $90^{\circ} \mathrm{C}$, sendo, nesta temperatura definidos dois tratamentos com durações de 3 e $6 \mathrm{~h}$.

Os tratamentos térmicos foram realizados em estufa com fluxo de ar contínuo, marca Fanem, modelo 330, utilizando-se de uma câmara para cada temperatura. Após os tratamentos, as sementes foram submetidas a testes para avaliar o efeito sobre o controle de $F$. oxysporum e na germinação das sementes.

Deteç̧ão de $\boldsymbol{F}$. oxysporum em sementes: As sementes submetidas aos tratamentos foram analisadas quanto à incidência de $F$. oxysporum pelo método de plaqueamento em papel de filtro ("Blotter Test") (ISTA, 1976), como segue: as sementes foram colocadas em caixas plásticas, tipo "gerbox", que medem 10x10x4 cm (comprimento x largura $\mathrm{x}$ altura), contendo duas folhas de papel de filtro umedecidas com água destilada estéril, sendo colocadas 50 sementes por "gerbox". As sementes foram incubadas por 10 dias, a $20 \pm 2$ ${ }^{\circ} \mathrm{C}$, fotoperíodo de $12 \mathrm{~h}$ de luz NUV (Near Ultra Violet) e 12 $\mathrm{h}$ escuro. Os fungos foram identificados pelas características morfológicas observadas aos microscópios estereoscópio e óptico.

Determinação do poder germinativo das sementes: Foi realizado o teste de germinação conforme prescrito nas Regras de Análises de Sementes (Ministério da Agricultura e Reforma Agrária, 1992), exceto na quantidade de sementes, que foram utilizadas 200 ao invés de 400. Em cada "gerbox" foram colocadas 50 sementes que após distribuição foram incubadas em câmara com $100 \%$ de UR, temperatura a $28{ }^{\circ} \mathrm{C}$, sob luz fluorescente contínua. A germinação foi avaliada aos seis e 11 dias após o início do teste.

\section{Tratamento térmico úmido das sementes inoculadas com F. oxysporum}

Sementes de alfafa inoculadas com $F$. oxysporum, acondicionadas em sacos de tecido de "organdi", foram imersas em banho-maria com agitador, a temperatura de $40^{\circ} \mathrm{C}$, para o pré-tratamento por períodos 10, 20 e $30 \mathrm{~min}$, e imediatamente transferidas para outro banho-maria regulado para 50 ou $60{ }^{\circ} \mathrm{C}$, com agitador, para o tratamento, por períodos de 10, 20 e $30 \mathrm{~min}$, e, em seguida, foram resfriadas em água $\mathrm{a} \pm 22{ }^{\circ} \mathrm{C}$. Sementes sem tratamento foram mantidas como testemunha. Após os tratamentos as sementes foram avaliadas quanto à incidência de $F$. oxysporum e germinação, conforme metodologia descrita anteriormente.

\section{Tratamento químico em sementes de alfafa inoculadas com F. oxysporum}

Para efeito de comparação, as sementes de alfafa inoculadas com $F$. oxysporum foram submetidas ao método convencional de tratamento químico com os produtos benomyl, thiabendazole, iprodione e thiram, nas dosagens recomendadas 
pelos fabricantes e nas dosagens triplicadas recomendadas. A avaliação dos tratamentos realizou-se por meio do teste de sanidade e germinação, descritos anteriormente.

\section{Delineamento experimental}

O delineamento experimental foi de blocos ao acaso com quatro repetições, onde a unidade experimental constituida de um "gerbox", contendo 50 sementes cada. Procedeu-se a análise de variância (ANOVA), aplicando-se o teste F $(5 \%)$ e, posteriormente, o teste Duncan $(5 \%)$ para verificar o efeito dos tratamentos na incidência de $F$. oxysporum e na germinação das sementes.

\section{RESULTADOS E DISCUSSÃO}

No presente trabalho não foi observado o desenvolvimento de $F$. oxysporum em sementes de alfafa, que foram submetidas ao tratamento térmico seco nas temperaturas de $60{ }^{\circ} \mathrm{C}$ (pré-tratamento) e $90{ }^{\circ} \mathrm{C}$ (tratamento), independente do tempo de exposição. Estes tratamentos também não afetaram significativamente a germinação das sementes (Tabela 1). $\mathrm{O}$ tratamento térmico seco $\left(60{ }^{\circ} \mathrm{C}\right.$ por $6 \mathrm{~h}$, seguido de $3 \mathrm{~h}$ a $\left.90{ }^{\circ} \mathrm{C}\right)$ foi relatado anteriormente controlando C. kikuchii em sementes de soja (Fonseca et al., 1994).

O tratamento térmico úmido, com pré-tratamento a $40{ }^{\circ} \mathrm{C}$ por 10,20 ou $30 \mathrm{~min}$, seguido do tratamento a 60 ${ }^{\circ} \mathrm{C}$ pelo mesmo período de exposição, erradicou $F$. oxysporum das sementes de alfafa, porém reduziu significativamente a germinação das sementes. A aplicação deste tratamento apenas a $60{ }^{\circ} \mathrm{C}$ por 20 e 30 min reduziu significativamente a incidência de $F$. oxysporum sem prejudicar a germinação das sementes, sendo que no período de 20 min de exposição, a germinação foi significativamente maior do que na testemunha (Tabela 2). Estes resultados concordam com Winter et al. (1997) que utilizando calor úmido $\left(52{ }^{\circ} \mathrm{C}\right.$ por $\left.10 \mathrm{~min}\right)$ controlou Fusarium nivale Ces. ex Sacc. e Fusarium graminearum Schwabe em sementes de cereais, com Erley et al. (1997) que erradicou Fusarium moniliforme (Sheldon) de sementes de milho (Zea mays L.) com a aplicação de calor úmido (57 e 60 ${ }^{\circ} \mathrm{C}$ por $15 \mathrm{~min}$ ), e com Prabhu et al. (1982) que reduziu

TABELA 1 - Efeito do tratamento térmico seco (60 e 90 ${ }^{\circ}$ C) na germinação e no controle de Fusarium oxysporum em sementes de alfafa (Medicago sativa)

\begin{tabular}{|c|c|c|c|}
\hline \multicolumn{2}{|c|}{$\begin{array}{c}\text { Tempo de exposição }(h) / \\
\text { temperaturas }\end{array}$} & \multirow{2}{*}{$\begin{array}{c}\text { Germinação } \\
(\%)\end{array}$} & \multirow{2}{*}{$\begin{array}{c}\text { Incidência de } \\
\text { Fusarium oxysporum } \\
(\%)\end{array}$} \\
\hline $60^{\circ} \mathrm{C}$ & $90^{\circ} \mathrm{C}$ & & \\
\hline 3 & 3 & $82,0 \mathrm{a}^{1}$ & $0,3 \mathrm{a}$ \\
\hline 3 & 6 & 76,5 a & $0,3 \mathrm{a}$ \\
\hline 6 & 6 & 76,0 a & $0,3 \mathrm{a}$ \\
\hline 0 & 0 & $89,5 \mathrm{a}$ & $20 \mathrm{~b}$ \\
\hline
\end{tabular}

${ }^{1}$ Médias seguidas de mesmas letras não diferem entre si em nível de $5 \%$ de significância pelo teste Duncan.
TABELA 2 - Efeito do tratamento térmico úmido (40 e $\left.60{ }^{\circ} \mathrm{C}\right)$ na germinação e no controle de Fusarium oxysporum em sementes de alfafa (Medicago sativa)

\begin{tabular}{|c|c|c|c|c|}
\hline \multicolumn{2}{|c|}{$\begin{array}{c}\text { Tempo de exposição (min) / } \\
\text { temperaturas }\end{array}$} & \multirow{2}{*}{\multicolumn{2}{|c|}{$\begin{array}{l}\text { Germinação } \\
(\%)\end{array}$}} & \multirow{2}{*}{$\begin{array}{c}\text { Incidência de } \\
\text { Fusarium } \\
\text { oxysporum }(\%)\end{array}$} \\
\hline $40^{\circ} \mathrm{C}$ & $60^{\circ} \mathrm{C}$ & & & \\
\hline 0 & 20 & 76,0 & $a^{1}$ & $0,1 \quad \mathrm{a}$ \\
\hline 0 & 30 & 71,0 & $a b$ & 0,1 a \\
\hline 10 & 10 & 37,0 & $\mathrm{c}$ & 0,1 a \\
\hline 10 & 20 & 29,0 & d & 0,1 a \\
\hline 10 & 30 & 14,0 & $\mathrm{f}$ & 0,1 a \\
\hline 20 & 10 & 21,5 & $\mathrm{e}$ & 0,1 a \\
\hline 20 & 20 & 11,0 & gf & 0,1 a \\
\hline 30 & 10 & 13,0 & f & 0,1 a \\
\hline 30 & 30 & 5,50 & $\mathrm{~g}$ & 0,1 a \\
\hline 0 & 0 & 67,0 & $\mathrm{~b}$ & $20 \mathrm{~b}$ \\
\hline
\end{tabular}

${ }^{1}$ Médias seguidas de mesmas letras não diferem entre si em nível de $5 \%$ de significância pelo teste Duncan.

a contaminação das sementes de Leucaena Benth por fungos com o tratamento a $85{ }^{\circ} \mathrm{C}$ por $5 \mathrm{~min}$, sem comprometer a germinação das sementes. Observa-se que o tratamento térmico úmido foi empregado para diferentes espécies de Fusarium e pode ser considerado uma opção para o seu controle.

Os tratamentos térmicos úmidos a $50{ }^{\circ} \mathrm{C}(10$ e $20 \mathrm{~min})$ e a $40{ }^{\circ} \mathrm{C}$ (pré-tratamento) por 10 ou $20 \mathrm{~min}$, seguidos de tratamento a $50{ }^{\circ} \mathrm{C}$ pelo mesmo período, controlaram significativamente $F$. oxysporum das sementes de alfafa sem afetar significativamente a germinação das sementes. Quando se utilizou a temperatura de $50{ }^{\circ} \mathrm{C}$ no período de $30 \mathrm{~min}$, os tratamentos foram eficientes na erradicação do fungo, entretanto afetaram significativamente a germinação das sementes de alfafa (Tabela 3). Winter et al. (1997) alcançaram resultados semelhantes, eliminando completamente $F$. oxysporum de sementes de cereais ao utilizarem calor úmido a $60{ }^{\circ} \mathrm{C}$ por $20 \mathrm{~min}$, com perdas no poder germinativo, no entanto na temperatura de $56-58{ }^{\circ} \mathrm{C}$ por $20 \mathrm{~min}$, o fungo foi controlado sem prejudicar a germinação.

Desta forma pode-se afirmar que, o sucesso do tratamento térmico depende do tipo de calor, seco ou úmido, da uniformidade da aplicação do calor, do período de exposição e da temperatura empregada.

Os fungicidas testados, benomyl, thiabendazole, iprodione e thiram, aumentaram a germinação das sementes de alfafa contaminadas com $F$. oxysporum, sendo que o produto benomyl aplicado nas dosagens de 50 e $150 \mathrm{~g}$ de i.a. por $\mathrm{Kg}$ de sementes e thiram aplicado na dosagem triplicada (210 g de i.a. por kg de sementes) aumentaram significativamente a germinação das sementes (Tabela 4$)$.

Benomyl, nas duas dosagens testadas (50 e $150 \mathrm{~g}$ de i.a. por $\mathrm{kg}$ de sementes) e thiabendazole na dosagem triplicada (60 g de i.a. por $\mathrm{kg}$ de sementes) erradicaram o fungo das sementes de alfafa (Tabela 4). Benomyl e thiabendazole foram descritos em literatura controlando $F$. oxysporum em sementes de outras culturas (Abd Elrazik et 
Erradicação de Fusarium oxysporum em sementes de alfafa utilizando termo...

TABELA 3 - Efeito do tratamento térmico úmido (40 e 50 $\left.{ }^{\circ} \mathrm{C}\right)$ na germinação e no controle de Fusarium oxysporum em sementes de alfafa (Medicago sativa)

\begin{tabular}{|c|c|c|c|c|}
\hline \multicolumn{2}{|c|}{$\begin{array}{c}\text { Tempo de exposição }(\mathrm{min}) / \\
\text { temperaturas }\left({ }^{\circ} \mathrm{C}\right)\end{array}$} & \multirow{2}{*}{\multicolumn{2}{|c|}{$\begin{array}{l}\text { Germinação } \\
(\%)\end{array}$}} & \multirow{2}{*}{$\begin{array}{c}\text { Incidência de } \\
\text { Fusarium oxysporum } \\
(\%)\end{array}$} \\
\hline $40^{\circ} \mathrm{C}$ & $50^{\circ} \mathrm{C}$ & & & \\
\hline 0 & 10 & 62,5 & $a^{1}$ & $1,75 \mathrm{a}$ \\
\hline 0 & 20 & 65,5 & $\mathrm{a}$ & $0,50 \mathrm{a}$ \\
\hline 0 & 30 & 43,5 & $\mathrm{~b}$ & 0,10 a \\
\hline 10 & 10 & 70,0 & $\mathrm{a}$ & $0,25 \mathrm{a}$ \\
\hline 10 & 20 & 67,0 & $\mathrm{a}$ & $1,25 \mathrm{a}$ \\
\hline 10 & 30 & 48,0 & $\mathrm{~b}$ & $0,10 \mathrm{a}$ \\
\hline 20 & 10 & 67,5 & $\mathrm{a}$ & $0,25 \mathrm{a}$ \\
\hline 20 & 20 & 55,0 & $\mathrm{ab}$ & $0,10 \mathrm{a}$ \\
\hline 20 & 30 & 40,0 & $\mathrm{~b}$ & 0,10 a \\
\hline 0 & 0 & 65,0 & $\mathrm{a}$ & $35,50 \mathrm{~b}$ \\
\hline
\end{tabular}

${ }^{1}$ Médias seguidas de mesmas letras não diferem entre si em nível de $5 \%$ de significância pelo teste Duncan.

TABELA 4 - Efeito de produtos químicos no poder germinativo e na sanidade de sementes de alfafa (Medicago sativa) contaminadas com Fusarium oxysporum

\begin{tabular}{|c|c|c|c|c|}
\hline \multicolumn{2}{|c|}{ TRATAMENTO } & \multirow{2}{*}{$\begin{array}{c}\text { Germinação } \\
(\%)\end{array}$} & \multirow{2}{*}{\multicolumn{2}{|c|}{$\begin{array}{c}\text { Incidência de } \\
\text { Fusarium } \\
\text { oxysporum }(\%)\end{array}$}} \\
\hline $\begin{array}{c}\text { Produto } \\
\text { Químico p.a.* }\end{array}$ & g. de i.a.** & & & \\
\hline Iprodione & 100 & 73,5 & 27,25 & $\mathrm{a}$ \\
\hline Iprodione & 300 & 78,5 & 17,00 & $b$ \\
\hline Thiram & 210 & 83,5 & 16,25 & $\mathrm{~b}$ \\
\hline Thiram & 630 & 76,0 & 5,75 & $\mathrm{c}$ \\
\hline Benomyl & 50 & 84,0 & 0,10 & $\mathrm{c}$ \\
\hline Benomyl & 150 & 88,0 & 0,10 & $\mathrm{c}$ \\
\hline Thiabendazole & 20 & 79,5 & 1,50 & $\mathrm{c}$ \\
\hline Thiabendazole & 60 & 74,5 & 0,10 & $\mathrm{c}$ \\
\hline Testemunha & & 68,0 & 22,75 & $a b$ \\
\hline
\end{tabular}

*p.a - princípio ativo

**i.a. (ingrediente ativo) - dosagem aplicada por $100 \mathrm{~kg}$ de sementes.

${ }^{1}$ Médias seguidas de mesmas letras não diferem entre si em nível de $5 \%$ de significância pelo teste Duncan.

al., 1990; Lee, 1984). Em solução com acetona e 0,1\% de hipoclorito de sódio, benomyl erradicou $F$. oxysporum e outros contaminantes de sementes de aspargo (Aspargus officinalis L.) (Elmer \& Stephens, 1988). Embora estes fungicidas sejam eficientes na redução de espécies de $F$. oxysporum, alguns isolados apresentaram resistência a thiabendazole (Hanson et al., 1996) e a benomyl (Santos et al., 1999), demonstrando a necessidade da utilização de formas alternativas para o controle deste fungo.

Thiabendazole, na dosagem recomendada pelo fabricante e thiram, nas duas dosagens testadas, reduziram significativamente o fungo (Tabela 4). Estes resultados concordam com Gupta et al. (1997) que demonstraram a eficiência de thiram no controle de $F$. oxysporum em sementes de grão-de-bico (Cicer arietinum L.). Iprodione foi o único produto que não controlou significativamente $F$. oxysporum em sementes de alfafa nas duas dosagens testadas (100 e 300 $\mathrm{g}$ de i.a. por kg de sementes) (Tabela 4).

\section{AGRADECIMENTOS}

Os autores agradecem a colaboração de Murillo Lobo Junior, Alaíde Soares de Oliveira e de Maria de Fátima Batista pelo auxílio na realização deste trabalho

\section{REFERÊNCIAS BIBLIOGRÁFICAS}

ABD-ELRAZIK, A.A.. FAHMY, F.G., AMEIN, A.M. \& ELAMEIN, A.I. Role of onion seeds in transmission of damping-off causal fungi and chemical control of the disease. Assiut Journal of Agricultural Sciences 21:173193. 1990.

BOTTCHER, I. \& HORN, G. Investigations for destroying the seed-borne black leg fungus of sugarbeet, Phoma betae Frank (teleomorph Pleospora bjoerlingii Byford), by heat treatment. Archives of Phytopathology and Plant Protection 28:39-42. 1992.

DHIRENDRA, M., CHATURVEDI, R.V., TRIPATHI, S.C. $\&$ MISHRA, D. The fungitoxic effect of the essencial oil of the herb Nardostachys jatamansi DC. Tropical Agriculture 72:48-52. 1995.

DE LEON, C. \& GRUDLOYMA, U. Heat therapy of maize seed and its effect on viability. Indian Phytopathology 47:1. 1994.

DONALD, D.G.M. \& LUNDQUIST, J.E. Treatment of Eucalyptus seed to maximise germination. South African Forestry Journal 149:9-15. 1988.

ELLANKAYA, I.A., SOROLOVA, E.V. \& KURCHENKO, I.N. Occurrence of pathogenic fungi on creeping alfafa under forest-steppe zone conditions in Ukraine. Mikrobiologicheskii Zchurnal 58:32-38. 1995.

ELMER, W. H. \& STEPHENS, C. P. Comparison of technique for eliminating contaminants from asparagus seeds. Hort Science 23:1031-1032. 1988.

ERLEY, D.P., MYCOCK, D.J. \& BERJAK, P. The elimination of Fusarium moniliforme Sheldon infection in maize caryopses by hot water treatments. Seed Science and Technology 25:485-501. 1997.

FONSECA, J.N.L., MENDES, M.A.S., PINHEIRO, F.P. \& VIDAL, A.S. Efeito da termo e quimioterapia em sementes de soja contaminadas com Cercospora kikuchii. Fitopatologia brasileira 19:297. 1994. (Resumo).

GARRIDO, L.A., OLIVEIRA, A.S., VIDAL, A.S., MENDES, M.A.S. \& FONSECA, J.N.L. Sementes de Glycine max infetadas artificialmente com Phomopsis phaseoli $\mathrm{f}$. sp. meridionalis e Colletotrichum truncatum. Fitopatologia brasileira 21:359. 1996 (Resumo).

GUPTA, S.K., UPADHYAY, J.P. \& OJHA, K.L. Effect of fungicidal seed treatment on the incidence of chickpea wilt complex. Annals of Plant Protection Sciences 5(2):184-187. 1997.

HANSON, L.E., SCHWAGER, S.J. \& LORIA, R. Sensitivity to thiabendazole in Fusarium species associated with dry rot of potato. Phytopathology 86:378-384. 1996.

INTERNATIONAL SEED TESTING ASSOCIATION 
(ISTA). Seed health testing. Seed Science \& Technology 4:3-49. 1976.

KAUR, N.P. \& MUKHOPADHYAY, A.N. Integrated control of "chickpea wilt complex" by Trichoderma and chemical methods in India. Tropical Pest Management 38:372375.1992 .

LEE, D.H. Fungi associated with soybean seed, their pathogenicity and seed treatment. Korean Journal of Mycology 12:27-33. 1984.

MINISTÉRIO DA AGRICULTURA E REFORMA AGRÁRIA, Secretaria Nacional de Defesa Vegetal, Coordenação do Laboratório Vegetal, Programa Brasileiro de Qualidade e Produtividade, PBQP, Regras para Análise de Sementes, Brasília. 1992.

ORLIEZ-LUTHARDT, A. On the transfer of Fusarium wilt by seeds in China aster. Seed Science and Technology 26:67-76. 1998.

PRABHU, M.S.C., VENKATASUBBAIAH, P., SAFEEULLA, K.M., SHETTY, H.S. \& SATISHCHANDRA-PRABHU, M. Fungi associated with Leucaena seeds and their influence on germination. Annals of Tropical Research 4:151-155. 1982.

PRYOR, B.M., DAVIS, R.M. \& GILBERTSON, R.L. Detection and eradication of Alternaria radicina on carnot seed. Plant Disease 78:452-456. 1994.

RAJU, U.J. \& SIVAPRAKASAM, K. Influence of seed treatment with fungicides, bactericide, hot water and antagonists on the seedling vigour of cabbage. Madras Agricultural Journal 76:26-30. 1989.

RANDHAWA, H.S. \& AULAKH, K.S. Efficacy of hot water treatment to control seed-borne fungi of raya (Brassica juncea Com.). Indian Journal of Plant Pathology 4:7376. 1984.

REIS, A., OLIVEIRA, S.M.A. de, MENEZES, M., MARIANO, R. de L.R. \& de OLIVEIRA, S.M.A. Potencial de isolados de Trichoderma para biocontrole da murcha de Fusarium do feijoeiro. Summa Phytopathologica 21:16-20. 1995.

SANTOS, B.A., ZAMBOLIM, L., VENTURA, J.A. \& VALE, F.X.R. Resistência de Fusarium subglutinans f. sp. ananas ao benomyl. Fitopatologia brasileira 24:436-439. 1999.

VENKATASUBBAIAH, P., PRABHU, M.S.C., SHETTY, H.S. \& SAFEEULLA, K.M. Effect of hot water and chemical seed treatment on seed mycoflora in koo-babul. Bangladesh-Journal of Botany 13:121-129. 1984.

VIDHYASEKARAN, P. \& MUTHAMILAN, M. Development of formulations of Pseudomonas fluorescens for control of chickpea wilt. Plant Disease 79:782-786. 1995.

WINTER, W., BANZIGER, I., KREBS, H. \& RUEGGER, A. Water treatments against dampring-off diseases of cereals. Agrarforschung 4:467-470. 1997. 